Mario Bronfman 1

Jorge Díaz Polanco 2

\section{La Cooperación Técnica Internacional y las políticas de salud}

\author{
The International Technical Cooperation \\ and the health policies
}

Abstract This paper analyses the changes in ITC that can be endorsed to modifications in the international scenario as well as those that can be attributed to the functioning of the international organizations themselves. $\mathrm{H}$ ealth policies and health reform are taken as the central instances for the analysis, specially the role played by the W orld $\mathrm{H}$ ealth Organization and its area office, the Panamerican H ealth O rganization and its relationships with multilateral agencies. It also compares the outputs of ITC in terms of cost/effectiveness and, finally, it suggests courses of action addressed to improve the ITC, based upon three main aspects: consequences of the international scenario dynamics on the ITC, varieties of ITC, and the values that must steer the process of producing ITC. Key words International Technical Cooperation, $\mathrm{H}$ ealth policies, $\mathrm{H}$ ealth reform
Resumen Este artículo analiza los cambios ocurridos en la CTI atribuibles a dos procesos: los cambios en los escenarios internacionales y otros que surgen de las propias organizaciones que brindan CTI. Se toman dos procesos como casos centrales: las políticas y las reformas de salud, especialmenteel papel desempeñado por la Organización M undial de la Salud y su oficina para las A méricas, la O rganización Panamericana de la Salud, y sus relaciones con las agencias multilaterales. Compara los resultados de la CTI en términos de costo/ efectividad $y$, finalmente, sugiere opciones para mejorar el proceso de CTI basadas en las consecuencias de los cambios en los escenarios internacionales, las modalidades de CTI y los valores que deben orientar la producción de la CTI.

Palabras-claves Cooperación técnica internacional, Políticas de salud, Reforma de salud
1 Centro de Investigación en Sistemas de Salud del Instituto Nacional de Salud Pública. Av. Universidad 655, colonia Santa M aría Ahuacatitlán, 62508, Cuernavaca, M orelos, M éxico.

bronfman@insp3.insp.mx 2 Centro de Estudios del Desarrollo. Universidad, Central de Venezuela. 


\section{Introducción}

La cooperación internacional se desarrollo junto con las instituciones que crearon la comunidad de naciones. M uchos esfuerzos internacionales, bilaterales y multilaterales pueden identificarse antes de que finalizara la II Guerra M undial, sin embargo, la cooperación técnica que hoy conocemos debe gran parte de sus atributos y características al orden internacional inaugurado en las postrimerías de la última guerra mundial. La Cooperación Técnica Internacional (CTI) ha sido y continúa siendo uno delos principales mecanismos diseñados por la comunidad internacional para enfrentar los retos de un orden internacional que ha crecido en interdependencia e integración. Las tendencias que describen la evolución de la cooperación técnica en gran medida fueron resultado de las tensiones internacionales, de manera particular allí se expresaron los grandes conflictos de la segunda mitad del siglo 20 como el enfrentamiento Este-O este, la emergencia de nuevos Estados independientes que dio lugar a la descolonización y a los desiguales patrones de desarrollo social entre las diferentes regiones del mundo.

Sin embargo, los parámetros que guiaron la cooperación internacional durante casi cuarenta años ya no cumplen el mismo papel. En ese sentido, este trabajo propone que la Cooperación Técnica Internacional debe enfrentar el imperativo de modificar sus prácticas, orientaciones, contenidosy formas de organización con el fin de adaptarse a las nuevas circunstancias $y$, sobre todo, redefinir su articulación como componente de apoyo a las políticas sociales en los países objeto de la CTI, especialmente en el caso de salud. Esta reformulación, afortunadamente para el caso de América Latina y especialmente para el sector salud, se lleva a cabo en el marco de una importante tradición de cooperación técnica entre los países de la región (cooperación horizontal) y con otros países y organismos internacionales (principalmente cooperación norte-sur). M uchos países de la región cuentan con instituciones sólidas, sus gobiernos perciben a la cooperación técnica como un instrumento necesario para impulsar el desarrollo de la región y actualmente se han consolidado distintos proyectos de integración subregional que sin duda favorecerán las relaciones de cooperación entre los gobiernos y otros actores relevantes. Sin embargo, hay países en los cuales la institucionalidad es frágil o ha experimentado importantes daños. En estos casos las prioridades están dirigidas hacia objetivos políticos internos, frente a los cuales la CTI no necesariamente ocupa un lugar prominente y muchas veces es entendida como una rémora del neocolonialismo. Venezuela es uno de los casos emblemáticos.

Los cambios en la cooperación técnica internacional, sin embargo, no pueden atribuirse exclusivamente a las tran sformaciones del escenario internacional. La evolución misma de esta práctica encontró barreras originadas en su propia lógica de funcionamiento y organización. La segunda hipótesis que propongo sostiene que factores ligados con la organización, la lógica de funcionamiento, las prioridades y los contenidos de la cooperación técnica internacional obstaculizaron el desenvolvimiento de la misma. Los instrumentos que una vez fueron percibidos como apropiados para disminuir las desi gualdades entre países hoy se analizan a la luz de los resultados. El verticalismo, la burocratización, la el evada especialización, la escasa participación, la ausencia de mecanismos de rendición de cuentas y evaluación son algunos de los factores que hoy se considera disminuyeron el impacto de la cooperación internacional.

La crítica al paradigma tradicional, sin embargo, no se tradujo en el advenimiento de un model o que concentrara el consenso de todos los que participan en este campo. Los años noventa fueron de experimentación, se ensayaron nuevas formas de organización de la cooperación, las prioridades fueron reformuladas, nuevos actores emergieron como protagonistas, y todo esto ocurrió, además, en un clima internacional que coloca el tema de la pobreza en un lugar prominente de la agenda internacional (W orld Bank, 2001; WHO, 1999).

Por supuesto, también se han levantado dudas acerca de ia efectividad de la cooperación internacional, se ha preguntado si los logros han estado a la altura de los esfuerzos financieros de la comunidad internacional o si la ayuda debe dirigirse selectivamente hacia aquellos países que muestran un compromiso efectivo con las prioridades en materia de pobreza (Gwin et al., 1997). Estos cuestionamientos han estado acompañados por un cada vez menor compromiso financiero por parte de los países centrales. Esta tendencia puede atribuirse en parte a que el fin de la guerra fría ya no demanda la inversión de grandes recursos en la tarea de mostrar la superioridad de occidente, también se ha señalado que las relaciones de cooperación están sometidas a una suerte de fatiga de la ayuda 
(aid fatigue) provocada por los escasos resultados de la ayuda internacional (World Bank, 2001). Por otra parte, la tendencia a medir la CTI por la magnitud de los recursos invertidos es una práctica que debe ser desechada, por su dudosa confiabilidad, tal y como ha sido mostrado por algunos autores en el caso de Africa (Ferguson, 1994).

Ante estas tendencias contrastantes es necesario abocarse con mayor vigor a diseña las características y el perfil de la cooperación técnica en salud que la región necesita. En la primera parte describimos los cambios en el escenario internacional y se identifican las consecuencias específicas para la cooperación técnica. En la segunda parte, analizamos con mayor detalle la evolución reciente de la CTI distinguiendo entre sus modalidades. En la tercera parte se aborda la cuestión de los valores que debieran orientar la CTI. La cooperación no debiera limitar su campo de acción a lo puramente instrumental; la separación entre lo técnico y lo normativo es artificial y no refleja la dinámica real de los procesos sociales. Toda técnica conlleva definiciones axiológicas que es mejor hacer explícitas y se trata, justamente, de cómo rearticular estos componentes presentes en toda forma de CTI. Por último, sugiero al gunos de los temas que considero fundamentales para la discusión sobre el funcionamiento de la cooperación internacional.

\section{Cambios en el escenario internacional}

La coordinación de esfuerzos en el ámbito internacional no hubiera podido madurar sin el desarrollo de las instituciones internacionales que emergieron con el orden institucional de la posguerra. Sobrela evolución de la Cooperación Técnica Internacional (CTI) repercutieron los mismos factores que determinaron los cambios de las relaciones internacionales durante los últimos años. En los años ochenta, pero especialmente durante los noventa, las bases institucionales de la CTI experimentaron cambios sustantivos que alteraron los patrones tradicionales que habían organizado la CTI. Entre los factores que mayor impacto tuvieron podemos señalar a) el tránsito de un orden internacional a otro global; b) la redefinición de la relación entre estados, sociedad y mercado; y c) la emergencia de nuevos actores internacionales. La $\mathrm{CTI}$ en salud además enfrenta el reto de transformaciones más profundas, el perfil epidemio- lógico de la población en la región combina elementos de tipo tradicional y moderno lo cual conlleva a una demanda de servicios de salud compleja y dinámica a la vez.

\section{Globalización y salud: una relación incierta}

¿Por qué es relevante el tema de la globalización para la salud? Por dos razones. Primero, porque a ella están asociados cambios profundos en las relaciones internacionales y ello afecta el desenvolvimiento de las instituciones internacionales y regionales de salud. Segundo, porque los procesos sociales y económicos asociados con la globalización conllevan nuevos riesgos que es necesario abordar desde una perspectiva global.

Con respecto a la primera dimensión habría que señalar que la globalización ha modificado profundamente el escenario de la CTI. Esta emergió en un contexto donde primaban las fuerzas de la internacionalización y un orden construido básicamente en torno a la soberanía de los Estados nacionales. Aunque la globalización ha sido frecuentemente asociada, y a veces reducida, a los cambios tecnológicos y a la reorganización de la economía mundial, lo que realmente distingue a esta etapa de otras es la constitución de un orden relativamente autónomo de actores globales. El escenario internacional ya no es el campo exclusivo de acción de los Estados nacionales, sino que cada vez más debe entendérselo como un campo donde éstos participan en relaciones de cooperación y conflicto con otros actores (empresas transnacionales, organizaciones no gubernamentales, organismos mundiales y regionales).

Además, se asiste a la constitución de una nueva cultura global con contenidos contradictorios y ambivalentes que lo mismo favorecen la lucha contra la pobreza o impiden elevar las contribuciones de los países de mayores ingreso para apoyar el desarrollo de los de menor ingreso. Indudablemente que se han consolidado condiciones que favorecen muy particularmente a la $\mathrm{CTI}$, pero también han emergido otros elementos que la desalientan. Estos elementos "desalentadores" tienen que ver, entre otras cosas, con la paulatina reducción de la presencia de la sociedad en los procesos de toma de decisiones. En efecto, frente a la nueva y compleja trama de relaciones que se ha generado, la representatividad de los organismos globalizados queda en entredicho frente a las demandas de dicha sociedad. 
Los sistemas nacionales de salud no están en condiciones de enfrentar los retos que la globalización plantea en el campo de la salud, pero las instituciones internacionales tampoco han ofrecido respuestas porque los cambios han avanzado más rápido que su capacidad de adaptación (Frenk et al., 1997). La OM S y el Banco $M$ undial, entre otras organizaciones multilaterales, han acusado seriamente el impacto de los cambios en el escenario internacional, han tenido que revisar sus objetivos, estructuras de gobierno y mecanismos de coordinación ante las evidentes dificultades que ofrece en nuevo ambiente internacional (Frenk et al., 1997; Abbasi, 1999). La OM S y sus organizaciones regionales tendrán que desarrollar las formas institucionales y organizativas más adecuadas al nuevo escenario internacional y rediseñar la división del trabajo (y las responsabilidades) entre la OM S, los estados y las demás instituciones multilaterales, especialmente el Banco M undial.

La cuestión de la dimensión internacional de la salud no es un tema nuevo. Según Berlinguer (s.f.) puede identificarse empíricamente una globalización de las enfermedades a partir de 1492, aunque no es sino hasta finares del siglo 19 que se manifiestan los primeros intentos para ofrecer una respuesta internacional. La diferencia entre la actual coyuntura y las experiencias anteriores es que la globalización en tanto proceso adquiere una creciente autonomía que la al eja del control de los actores del escenario internacional, entre ellos los Estados. Para algunos, el mayor peligro asociado con la globalización proviene del debilitamiento de las fronteras nacionales y las consecuentes condiciones favorables para una rápida difusión de enfermedades contagiosas. A ello habría que agregar los efectos derivados de la constante presión sobre los recursos naturales y los cambios en la nutrición derivados de la universalización de patrones de consumo poco saludables (D aulaire, 1999). La globalización, para otros observadores, es sinónimo de expansión de los mercados y debilitamiento de las instituciones, los sistemas nacionales de salud, especialmente en el caso de los países de menor desarrollo, se verían afectados negativamente por el debilitamiento de la cooperación internacional y la mayor exposición a las fuerzas del mercado ( $\mathrm{Ne}$ siah, 1999).

Todos han advertido sobre las consecuencias de la globalización sobre el funcionamiento de los sistemas de salud y la emergencia de nuevos perfiles epidemiológicos, sin embargo, las relaciones más específicas y la dirección de las mismas están sujetas a debate. En ese sentido resulta sugerente el modelo propuesto por Woodward (2001). Los autores distinguem efectos directos e indirectos entre el proceso de globalización y los sistemas de salud. Los efectos directos se expresan en el funcionamiento de los sistemas de salud y las políticas como resultado de la integración de los países a los acuerdos y mecanismos internacionales (por ejemplo, los mecanismos que regulan el comercio mundial). Los efectos indirectos sobre los sistemas de salud operan a través del funcionamiento de las economías nacionales. Esteúltimo punto es crucial teniendo en cuenta los efectos sociales provocados por las reformas estructurales y la apertura de las economías a la competencia internacional. En todo caso es importante identificar las dos aristas del proceso; por un lado, la multiplicación de factores que vulneran el bienestar y la salud de la población y, por otro, la exploración de nuevos mecanismos que permitan revertir, anticipar y atender los efectos negativos.

\section{Redefínición del papel del Estado}

El Estado como institución y especialmente sus funciones sociales y económicas, fueron sometidos a un duro cuestionamiento durante los años ochenta y noventa. El movimiento neo-conservador cuestionó profundamente los model os de desarrollo que se basaban en un alto involucramiento de las instituciones públicas en la economia y la sociedad y convirtió al mercado en el único mecanismo capaz de impulsar un proceso sostenido de crecimiento econômico. Obviamente, una de las consecuencias fue el cuestionamiento del papel de las instituciones públicas como medios de transformación de las condiciones sociales. En su lugar el mercado fue definido además como el instrumento más adecuado y eficiente para al canzar las metas de justicia y equidad. La parcial retirada de los Estados dio lugar a la emergencia de una gran variedad de actores, la CTI ya no volvería a estar organizada exclusivamente en torno a los Estados, paulatinamente las ON G, las grandes trasnacionales, los organismos multilaterales y bilaterales y los mecanismos de coordinación regionales adquirirían un mayor protagonismo.

En la perspectiva de la CTI es importante distinguir que el debilitamiento del estado ha ocurrido en dos frentes. En el interior de los espacios nacionales los Estados perdieron terreno 
ante la expansión de los mercados y el fortalecimiento de los actores de la sociedad civil, en el plano internacional los Estados también cedieron terreno ante actores global es como los organismos multilaterales que crecieron en autonomía y otros actores que emergieron con el proceso mismo de globalización, como la industria farmacéutica en el caso del sector salud. Esto no quiere decir que la figura del Estado nacional haya desaparecido o que ya no gravite en el plano internacional. Lo que ha sucedido es que las bases tradicionales de poder del Estado se han erosionado y ello ha provocado un cambio en las relaciones de poder a nivel internacional.

Es importante advertir que la constitución de un orden internacional no sólo se sostiene en el fortalecimiento o aparición de nuevos actores. Un elemento distintivo es la constitución de un orden jurídico internacional al cual paulatinamente se han ido incorporando los Estados. La formación de bloques regionales ha sido una de las respuestas más comunes, la Unión Europea, el TLC en N orteamérica, el M ercosur en Sudamérica y otras experiencias similares en Asia indican que los bloques regionales se han constituido en los ámbitos donde actual mente se negocian cuestiones relevantes no sólo para la economía, sino también para las políticas sociales. Cualquier estrategia de CTI deberá contar en el futuro con una clara inserción en estos espacios regionales.

\section{Los retos de las instituciones sanitarias internacionales}

La Organización M undial de la Salud es una institución líder a nivel mundial. La experiencia acumulada en asuntos normativos y asistencia técnica la convierten en la más importante autoridad técnica en salud en el ámbito internacional. Sin embargo, distintos observadores han advertido sobre las dificultades que O M S enfrenta para ajustarse a los cambios del entorno internacional. Algunos recomiendan una revisión de las tareas estratégicas para identificar las áreas donde las actividades de la OM S son complementarias y donde existe yuxtaposición o competencia con las agendas de otros organismos con el objetivo de definir más claramente los campos de acción y mejorar la coordinación (Frenk et al., 1997). Otros han señalado quela OMS ha renunciado deliberadamente a asumir el liderazgo en el campo de la salud internacional para evitar conflictos y disputas con otros actores globales. Como resultado de ello la or- ganización se ha refugiado en sus tareas técnicas y otros actores han asumido esterol político (Godlee, 1994). Las críticas, por otro lado, también han señalado que la organización tiene un compromiso cada vez más débil con los valores de la equidad y justicia ( $N$ avarro, 2001).

Un cambio que modificó el escenario político de la salud a nivel internacional durante los noventa fue el creciente liderazgo del Banco M undial en el campo de la salud. Varios factores confluyeron en este proceso. Por un lado, la OM S adolecía de serios problemas organizativos, tenía una excesiva burocratización y centralización; los recursos económicos eran insuficientes para cumplir sus objetivos; ella manifestaba problemas de adaptación a la nueva situación de la salud en el mundo que requería de nuevas estrategias multidisciplinarias y los vínculos con las instancias gubernamentales no directamente ligadas con la salud eran muy débiles Las inercias organizativas habían adquirido tal peso que estaban afectando seriamente la capacidad de respuesta de la organización a un medio sometido a fuertes transformaciones.

Estas razones explican por quê la OM S dejó de asumir ciertas responsabilidades políticas a nivel internacional, pero no son suficientes para entender cómo y por que Banco M undial llegó ocupar este espacio con tanta facilidad, sobre todo teniendo en cuenta que el tema salud no había formado parte de su agenda hasta mediados de los ochenta (World Bank, 1999). Dos son las razones: por un lado, la importancia de los sistemas de seguridad social y de la salud pública en los presupuestos nacionales. El déficit fiscal de los gobiernos y las condiciones de austeridad de las economías nacionales justificaron la implementación de reformas estructurales del sector. Por otro lado, el tema de la pobreza comenzó a convertirse en el eje de acción del banco, en este proceso la salud y la educación, en tanto componentes fundamentales del capital humano y social, se convirtieron en los sectores estratégicos de las políticas dirigidas a erradicar la pobreza, en un contexto en el cual el predominio de la idea de mercado, como regulador de la vida social, había adquirido una cierta preeminencia.

La salud se ha convertido en un tópico vinculado estrechamente con variables de carácter económico y ésta tiene a su vez una incidencia en el desempeño de las economías. Así mismo, el mejoramiento de la salud está asociado con problemas, políticas y programas en otras áreas: educación, medio ambiente, empleo, urbaniza- 
ción. En la medida en que el BM y las instituciones financieras multilaterales se han involucrado en programas de reforma en distintos sectores (trabajo, educación, educación superior) están en una mejor posición para construir los vínculos sectoriales más relevantes para el funcionamiento del sector salud. Un aporte sustantivo del BM es que ha contribuido a consolidar una visión más integral de la salud, estableciendo vínculos con otros fenómenos sociales (empleo, pobreza) y políticos (educación)

El enfoque del BM constituye un serio reto para reconstituir el liderazgo de la OM S. Este proceso pasa indudablemente por reconocer los aciertos del enfoque del Banco M undial, pero también por elaborar una perspectiva propia que permita superar las limitaciones que caracterizam al enfoque del BM. Aunque aqui sejuegan definiciones estratégicas, un campo donde pueden materializarse estas reformas es en el ámbito de la CTI en salud.

\section{Nuevos actores: los retos de la coordinación en un contexto inestable}

El reporte de la Conferencia Internacional sobre Investigación en Salud para el Desarrollo destaca que una de las características distintivas del nuevo escenario internacional es el creciente número de actores, la diversidad de estrategias que ellos impulsan y el creciente peso que está adquiriendo el sector privado (IOC, 2000). El orden internacional emergente, se ha señalado, tiene fisuras estructurales muy diferentes a las conocidas en el contexto de la guerra fria. Por supuesto, la existencia de estas barreras, que separan regiones, países y grupos sociales, no deja de ser una paradoja para una época dominada por la revolución en las comunicaciones y el transporte.

El escenario internacional está dominado por actores que han concentrado importantes cuotas de poder merced al reflujo de los estados nacionales. Aunque existen organismos que han logrado convertirse en autoridad en ciertos campos, cierto es también que han surgido nuevos actores con recursos y márgenes de acción muy amplios (Vg.: fundaciones privadas). Si la coordinación entre los organismos prestadores de cooperación técnica y las instituciones receptaras era problemática, ésta tarea es hoy más compleja debido al mayor número de actores que se desenvuelven en el escenario internacional. Aumentar la coordinación, discriminar campos de acción y establecer agendas comunes son algu- nos de los retos que los organismos internacionales prestadores de servicio deben resolver. La experiencia reciente ha mostrado que no es factible aspirar a consensos que involucren a todos los actores que operan en el campo de la cooperación técnica; por el contrario, las modalidades predominantes consisten en alianzas específicas entre actores alrededor de problemas puntuales. Las iniciativas impulsadas por la OMS y diversos actores (fundaciones, empresas farmacéuticas, gobiernos, etc.) en torno a distintos problemas (malaria, VIH/SIDA, vacunas, tuberculosis) se corresponden con esta modalidad emergente de colaboración (Widdus, 2001).

\section{Enfrentando los retos epidemiológicos en la región}

La transición demográfica y epidemiológica actualmente en curso plantea serios retos a las instituciones de salud de la región (Bobadilia et al., 1992). La transición demográfica se ha consolidado prácticamente en todos los países de América, la disminución constante de las tasas de mortalidad y fecundidad ha conducido a una notoria disminución del crecimiento demográfico, y esto a su vez, ha sentado las bases para un paulatino envejecimiento de la población. Aunque este patrón demográfico caracteriza a gran parte de la región, también es importante señalar que en muchos contextos sociales continúan prevaleciendo patrones demográficos tradicionales, especialmente allí donde predominan la marginalidad y la pobreza extrema. Así, las instituciones de salud en la región tienen que enfrentar todavia altas tasas de mortalidad y fecundidad en ciertas regiones y grupos sociales, mientras que las demandas de servicios de salud en las grandes ciudades asumen características total mente diferentes.

En las Américas, de igual modo, conviven viejos y nuevos problemas de salud, los procesos de modernización, urbanización y desarrollo económico dieron lugar a una nueva estructura de morbilidad y mortalidad en donde coexisten enfermedades características de contextos sociales de muy bajo desarrollo social y económico (infecciosas) con los padecimientos propios de las sociedades modernas (accidentes, cardio-vasculares). Los sistemas de salud enfrentan un serio reto de ofrecer respuestas para contexto sanitario muy heterogéneo y que a su vez está en pleno proceso de cambio. Por ejemplo, padecimientos como la malaria, la tuberculosis el dengue y el VIHISIDA continúan gravi- 
tando con gran fuerza en los niveles de morbilidad y mortalidad en regiones como América Central y Brasil (OPS, 2001).

\section{La experiencia de cooperación Sur-Sur en América}

La región de las Américas cuenta con una prolongada y fructífera historia de cooperación técnica. En primer lugar, es importante recordar que en 1978 se firma el Plan de Acción de Buenos Aires para Promover y Realizar la Cooperación Técnica entre los Países en Desarrollo, que posteriormente adoptaría la ONU como documento básico de la cooperación horizontal. La Cooperación Técnica entre Países en Desarrollo (CTPD) sentó las bases para un nuevo modelo de cooperación basado en las relaciones entre los países en desarrollo. Este modelo buscaba fomentar la capacidad de estos países para encontrar soluciones a sus problemas de crecimiento, reforzar el intercambio de experiencias e identificar y analizar los problemas comunes y aumentar las comunicaciones entre los países.

En el plano nacional el Plan de Buenos Aires recomienda crear los marcos normativos adecuados y las instituciones y organismos encargados de promover la CT horizontal, fortalecer los sistemas nacionales de información e investigación, promover el intercambio en materia de políticas, incluir actores no públicos a la CT (organizaciones profesionales, empresas, instituciones educación e investigación) y fomentar los vínculos bilaterales. En el plano subregional y regional el Plan propone crear y fortalecer las organizaciones de CT en el ámbito regional, identificar las oportunidades de CT entre los países en desarrollo, crear nuevos vínculos de cooperación entre los países en desarrollo, mejorar los sistemas de información re gionales y apoyar a los centros nacionales de investigación. En el plano mundial se propone fomentar la colaboración técnica, mejorar el flujo y generación de información, movilizar la capacidad del sistema de $\mathrm{N}$ aciones $\mathrm{U}$ nidas en materia de CT y comprometer a los países desarrollados en la CT. En este contexto se emprendieron numerosas iniciativas de cooperación impulsadas principalmente por los países más desarrollados de la región. Las modalidades más usuales de cooperación consistieron en el apoyo con expertos en diversas materias, la transferencia de tecnología de los países más desarrollados a los menos desarrollados y una variedad de medidas orientadas a fomentar el intercambio comercial (Pérez et al., 1998).

Uno de los logros más significativos de este enfoque en la región fue la creación de una serie de oficinas gubernamentales encargadas de coordinar la cooperación técnica. Sin embargo, la inestabilidad política y la persistencia de las crisis económicas afectaron el desenvolvimiento de los proyectos de cooperación técnica horizontal. La importancia estratégica de la cooperación horizontal se vio reflejada posteriormente en el interés puesto por diversos organismos regionales. El Banco Interamericano de Desarrollo, Ia CEPAL y la propia Organización de los Estados Americanos tienen diversos programas de cooperación horizontal.

La O rganización Panamericana de la Salud ha dado pasos significativos en el desarrollo de model os de cooperación horizontal. Ejemplo de ello es el proyecto Convergencia. El proyecto, entre otros objetivos, busca promover la generación de información, formación de recursos humanos, promover políticas de tecnología en salud y elaborar una metodología de cooperación técnica en salud (Panisset, 1992).

\section{H acia un modelo estratégico de cooperación técnica}

LaCTI vive un momento de transición. La crítica al modelo tradicional de cooperación técnica ha mostrado que muchos aspectos de ese modelo de cooperación influían negativamente en los resultados de la misma. Sin embargo, esto no ha dado lugar a la emergencia de un nuevo modelo de cooperación técnica. Por el contrario, la tendencia pareciera apuntar hacia un esquema pluralista, donde coexistirán diferentes perspectivas de cooperación técnica que se distinguirán por la forma en que combinen los aspectos clásicos e innovadores. Veamos cómo ha cambiado el enfoque en torno a los componentes de la CTI.

\section{Formación de agendas de cooperación. ¿Quién decideque?}

La definición de la agenda de cooperación constituía un ámbito donde claramente se expresaban las asimetrías entre prestadores y receptores de cooperación. El control de los recursos financieros y técnicos habilitaba a las instituciones que ofrecían CTI a ejercer una autoridad casi completa sobre el proceso de diseño 
del proyecto. Las instituciones receptaras de la cooperación por lo general se concentraban en los aspectos ligados con la implementación de los mismos. Este esquema de trabajo se sustentaba en gran medida en la idea de que los problemas biomédicos tenían soluciones de tipo técnico, por lo tanto, el control de los medios técnicos justificaba en gran medida la autoridad ejercida por las instituciones oferentes. M uchas veces el alto grado de especialización de los proyectos también contribuía a que las instituciones que ofrecían su colaboración técnica ejercieran un amplio dominio sobre la marcha del conjunto del proyecto. Este aspecto es especialmente importante cuando se observa cómo, en los proyectos de reforma del sector receptores de cooperación técnica, los temas centrales eran discutidos en los ministerios de finanzas, convirtiendo a los ministerios de salud en meros observadores y afianzando una visión economicista de los temas de la reforma.

Esta división del trabajo entre los actores de la cooperación técnica mostró limitaciones importantes en cuanto a los resultados obtenidos y el nível de compromiso promovido entre las instituciones receptaras de la cooperación técnica. En los procesos de reforma sectorial dicho compromiso se media como obligación financiera local y nacional. La primera crítica señaló la necesidad de que los proyectos de cooperación técnica se formularan teniendo como horizonte las prioridades establecidas por las instituciones locales de los países receptores de asistencia. Si se pretendía que los resultados de la CTI confluyeran con los esfuerzos nacionales en materia de desarrollo, éstos debían formularse con base a las orientaciones establecidas en cada campo en particular. Pero este paso era insuficiente, una articulación exitosa entre los objetivos de la CTI y las prioridades nacionales sólo podía fundarse en un firme compromiso por parte de los actores locales.

El Banco M undial en su más reciente informe reconoce que la participación de los Actores en todas las etapas de los proyectos constituye el más importante incentivo para obtener mayores grados de compromisos por parte de los actores locales (2001). El reto fundamental consiste entonces en coordinar los distintos enfoques que los actores (organismos internacionales, ONG, gobiernos, etc.) en distintas escalas (internacional, nacional, local) tienen sobre problemas similares. La Organización M undial de la Salud ha puntualizado esta estrategia muy claramente, la cooperación técnica debe orientarse hacia aque- llos países cuyos gobiernos han mostrado a través de sus políticas un compromiso con la salud de los más pobres y con el fortalecimiento y modernización de los sistemas de salud (WHO, 1999).

\section{Formación de recursos humanos}

En el modelo tradicional de cooperación técnica no existe una preocupación importante por la formación de recursos humanos en los países receptores de ayuda. En parte esto es así porque se partía del presupuesto de que la relación de cooperación estaba conformada por un actor que tenía los recursos humanos y técnicos y otro que carecía de los mismos (Costello et al., 2000; Jacobs, 1996). Incluso, en sus versiones más negativas, la cooperación al entaba la pérdida de valiosos recursos debido al reclutamiento de talentos por parte de los organismos y países oferentes de cooperación (Costello et al., 2000). Asimismo, la débil participación de las instituciones de investigación locales no alentaba el aprovechamiento de los recuros humanos locales.

La preocupación por la formación de recursos humanos en los países receptores de CTI creció conforme se advertía que los proyectos no eran sustentables en el largo plazo. En ese contexto es que se produjo un lento cambio hacia un esquema más integral que privilegia la utilización de recursos humanos locales, promovió la formación de redes institucionales integradas con los centros de investigación y desarrollo locales y, en general, se buscó establecer una relación más estrecha entre formación de recursos humanos y las prioridades sociales en los países receptores de asistencia técnica.

La cooperación internacional, especialmente la de tipo horizontal, tiene aqui una área importante de desarrollo y colaboración. La provisión de un servicio de salud eficiente y decalidad depende en un grado muy alto de los recursos humanos involucrados y los servicios de salud de la región tienen en este rubro un déficit muy importante. La formación de los profesionales no se orienta por las necesidades del sector, la productividad y losingresos de los trabajadores son bajos y en general las condiciones de trabajo suelen ser poco estimulantes ( $M$ artínez et al., 1999). La introducción de criterios de planeación estratégica de los recursos humanos en el sector salud constituye un paso fundamental, las instancias regionales de CTI podrían en ese sentido desarrollar modelos de gestión que podrían orientar las decisiones en el ámbi- 
to nacional. Por otro lado, la CTI podría cumprir un papel importante coordinando la difusión de las experiencias nacionales.

\section{Fortalecimiento institucional}

Por mucho tiempo predominó una visión de la cooperación muy restringida al evento de la transferencia de la asistencia. No se percibía que las características del contexto que rodeaba el proyecto, la red de relaciones institucionales que sostenía a los actores y las capacidades institucionalesjugaban un papel decisivo en los resultados de la cooperación. La revisión crítica de las experiencias de CTI mostraron que los resultados insatisfactorios estaban en muchos casos vinculados con debilidades de tipo institucional u organizativo que inhibían el desarrollo y maduración de los proyectos. Aspectos como la historia, la capacidad administrativa, el compromiso de los actores y el nivel de entrenamiento de los mismos fueron algunos de los factores que con mayor fuerza influían en los resultados de los proyectos (Jacobs, 1996).

Esto llevó a que los esfuerzos de la cooperación internacional desplazaran su interés desde los fines hacia los medios. La sustentabilidad de los proyectos en articulación con organismos claves a nivel internacional como la OM S enmarcados en la cooperación internacional ya no dependía exclusivamente de la destreza de los profesionales o de una correcta selección de técnicas y procedimientos. En parte las condiciones de éxito de los proyectos dependían ahora de la capacidad de las instituciones para absorber y poner en marcha los recursos materiales y humanos canalizados por la CTI. En este contexto, una parte del esfuerzo de cooperación tuvo que dirigirse a crear estas condiciones. Pero el reconocimiento del papel de las instituciones conoce también otras fuentes, durante los años noventa pudo percibirse un notable giro en el discurso de algunos organismos dominantes del escenario internacional, entre ellos el Banco M undial, que reconocieron explícitamente que el mercado no era la única alternativa para enfrentar los problemas sociales.

Sin embargo, junto con el reconocimiento otorgado al papel de las instituciones y las políticas también han aparecido formas nuevas de condicionar la ayuda financiera y técnica internacional (World Bank, 2001). La propuesta de focalizar la ayuda internacional en función de los ingresos per capita de un país y del grado de compromiso previo de sus instituciones públi- cas con los objetivos de la cooperación técnica modifica incluso los objetivos de la cooperación. La construcción de aquellas instituciones y políticas favorables para el desarrollo y la cooperación ya no es objetivo de la CTI sino una condición para su implementación.

Este giro, sin embargo, trae aparejados nuevos problemas para los países receptores y sus proyectos de política en el área de salud porque tiende a burocratizar los procesos, genera problemas institucionales e incrementa el tiempo de impacto de las políticas así disemadas (Díaz Polanco, 2001).

\section{Transferencia de tecnología}

El esquema tradicional de asistencia técnica funcionaba con base en una clara división del trabajo, por un lado, los actores que poseen el conocimiento y las técnicas necesarias para enfrentar determinados problemas y, por otro lado, los actores que tienen problemas pero que carecen de esos conocimientos y técnicas. La re lación asentada sobre esta desigual distribución de las necesidades y recursos dio origen a un esquema poco equitativo y en muchos casos ineficiente. Esta relación se veía reformada por la fuerza de las evidencias, efectivamente allí había un problema (la carencia) y también una solución apropiada (el dispositivo tecnológico). El fetichismo de la tecnología también promovía la errónea idea de que una misma solución tecnológica podía aplicarse a casos similares aunque en contextos sociales y culturales distintos. La crítica a este paradigma comenzó a señalar los problemas asociados a la falta de conexión entre la asistencia técnica y las prioridades en materia de salud en los países en desarrollo y la escasa vinculación de la CTI con el desarrollo de las capacidades de investigación en cada país.

La transferencia de tecnología entre instituciones de países desarrollados y en vías de desarrollo debe hacerse en el marco de las relaciones a largo plazo, la transferencia de tecnología debe ser apropiada a los diversos contextos sociales y culturales, las prioridades locales deben estar relevadas en los proyectos y programas de colaboración, el entrenamiento de cuadros y la investigación debe estar vinculada con las acciones sanitarias del país y la relación de cooperación N orte-Sur debe estar acompañada por el estímulo de las relaciones de cooperación horizontales ( $H$ arris et al., 2000).

La cooperación técnica internacional en las áreas de investigación y desarrollo, según la 
Conferencia sobre Investigación en Salud, debiera estar guiada por las siguientes orientaciones: a) la agenda de investigación en salud debe basarse en las prioridades nacionales aunque sin olvidar las interacciones regionales e internacionales; b) se requiere mejorar el ambiente de investigación en los países en desarrollo con el objetivo de desarrollar un efectivo sistema de ínvestigación; c) los países en vías de desarrollo debieran tener mayor visibilidad a nivel internacional; d) la investigación en salud debe estar vinculada con la agenda de desarrollo (IOC, 2000).

\section{Rendición de cuentas}

Los esquemas tradicionales de rendición de cuentas sólo contemplaban mecanismos de inspección y vigilancia de las instituciones receptoras de asistencia técnica. Excluidos de esta relación estaban las demás instituciones gubernamentales de los países receptores, los ciudadanos y otros organismos internacionales relevantes según la materia. Tomando en consideración las asi metrías que gobernaban estas relaciones, los mecanismos de rendición de cuentas funcionaban en los hechos como dispositivos de control. También habría que señalar que el objeto de la rendición de cuentas son las acciones enmarcadas en determinado proyectos y bajo la responsabilidad de las instituciones receptaras. De este modo, las instituciones que ofrecen su cooperación quedaban fuera de todo mecanismo de inspección y rendición de cuentas.

La crítica puso en evidencia que entre quienes ofrecían cooperación y quienes la recibían existían relaciones de poder. La desigual distribución de recursos favorecía a unos en detrimento de los otros. Sin embargo, la cuestión central no era el poder en sí mismo (la posesión de recursos) sino la ausencia de regulaciones orientadas a producir un intercambio más equilibrado entre los actores involucrados. La desigualdad no regulada era una fuerza poderosa que desalentaba el compromiso de los actores y empobrecía los resultados de la cooperación. La crítica, como puede apreciarse, tuvo el acierto político de identificar los aspectos no democráticos de la relación, pero también fue pragmática en la medida que identificó el vínculo entre este tipo de relación y los escasos resultados (H elleiner, 2000).

La rendición de cuentas es un instrumento que permite abrir a la discusión y debate los asuntos que son de interés público. En ese sen- tido existe un creciente interés por profundizar su utilización más allá de la actuación de los actores subordinados de la relación de cooperación técnica. Los organismos internacionales, las organizaciones no gubernamentales, en fin toda la gama de actores susceptibles de involucrarse en una relación de cooperación técnica, debe estar sujeta a algún mecanismo de rendición de cuentas que permita evaluar el desempeño de estos organismos en determinado país y sector. Un sistema de relaciones de cooperación técnica organizadas con base a un sistema de evaluación y monitoreo que involucre a todos los actores constituye una herramienta clave para promover alianzas antes que relaciones prestador-cliente. El seguimiento y la evaluación de procesos y resultados, inclusive de impacto, forma parte hoy dia de la formulación de proyectos que son objeto de CTI, pero requieren de la existencia de una cierta "cultura organizacional", no siempre presente en todos los países.

\section{La CTI y los procesos de reforma sectorial}

Los sistemas nacionales de salud en la región han conocido importantes reformas que abarcaron aspectos financieros, administrativos e institucionales. Estas transformaciones están construyendo nuevas bases para los sistemas de salud a largo plazo, en esta línea la CTI de la OPS podría desarrollar una línea de trabajo en el sentido de insertarse en estas relaciones de cooperación para contribuir con su opinión técnica y eventualmente evaluar y monitorear los resultados de los procesos de reforma. Esta es una cuestión crítica por varias razones: a) las reformas institucionales del sector salud (descentralización, administración, privatización) afectarán la calidad y nivel de provisión de los servicios de salud en el mediano y largo plazo, muchas veces estas reformas siguieron criterios presupuestarios y macroeconómicos antes que sanitarios por tal razón es perentorio estudiar los efectos de las reformas pasadas sobre la salud de la población (y sobre la pobreza y la desigualdad; b) otro papel importante de la asistencia técnica de la OPS estaría encaminado a evaluar el desempeño de los donors, que ha estado siempre fuera de toda jurisdicción nacional o regional, salvo la del país que ofrece ayuda; esta situación ha estimulado el establecimiento de relaciones desiguales, ineficiencias y desperdicio de recursos. LaCTI de la OPS podría en este sentido ofrecer a los donors y los países recepto- 
res de ayuda la opinión de un organismo altamente calificado en términos técnicos y políticamente independiente.

\section{Modelos de organización: jerarquía versus red}

La adaptabilidad a los cambios del entorno es un activo importante de las organizaciones que se desenvuel ven en contextos caracterizados por la Competencia. Como lo hemos sostenido, el agotamiento del modelo de cooperación tradicional en gran medida puede ser atribuido a la escasa capacidad de adaptación a las cambiantes condiciones del entorno. El reto más importante para la cooperación técnica en salud para las Américas consiste en diseñar el modelo de organización más apropiado para las tareas que desempeñará.

Los modelos tradicionales de asistencia técnica, como ya vimos, se distinguían por la jerarquización elos distintos niveles involucrados en la relación de asistencia técnica. El control de la agenda por parte de los oferentes de cooperación y el débil involucramiento de los actores receptores tendían a reproducir este esquema burocrático de organización. Otra característica distintiva era la escasa participación de actores distintos a los que ofertaban y recibían la asistencia técnica.

La idea de un modelo de organización más flexible nos coloca directamente en la perspectiva de la organización basada en redes. Siguiendo a Castells, las redes pueden definirse como conjuntos de nodos interconectados que pue den tener un principio jerárquico pero que definitivamente carecen de centro. La fortaleza de las redes reside en su flexibilidad, el carácter descentralizado de las actividades que la red contiene, la capacidad para absorber nuevas demandas y tareas sin introducir modificaciones sustanciales en las regias y objetivos de la organización (Castells, 1998). La organización por redes promueve el aprendizaje y el intercambio con el ambiente porque estimula el flujo de información a través de los vínculos y nodos.

El principal reto consiste en hacer compatible las formas actuales de organización de la cooperación técnica con la paulatina introducción de esquemas basados en redes. Ambas modalidades de organización tienen que pensarse como complementarias, el reto mayor consiste en buscar los mecanismos de articulación más apropiados para extraer los máximos beneficies de ambas modalidades.

\section{Solidaridad, equidad y eficiencia}

La Cooperación Técnica Internacional ya no puede desenvolverse en los estrechos limites de las soluciones técnicas. Esta alternativa impone altos costos a la cooperación porque inhibe el planteamiento de enfoques integrales y no reconoce la influencia de factores no técnicos culturales, políticos, sociales - en la constitución de los problemas que son objeto de la CTI. El repliegue en lo técnico, como ya vimos, fue en parte la actitud que asumió la OM S ante la incertidumbre de los anos ochenta y ello tuvo consecuencias en el plano del liderazgo mundial de esta institución. La idea de enfoques integrales en la CTI recupera también una idea que muchas veces se soslaya: las acciones y programas públicos comportan valores, aspiraciones y visiones del mundo que es necesario hacer explícito.

Los model os asistencialistas de cooperación sostuvieron que era posible aislar lo técnico de los elementos políticos y culturales involucrados en las relaciones entre los países, un intento vano por resguardar algo que se considerada "deseable y necesario" (la asistencia) contra aquello que se creía "contaminaba o impedía" los esfuerzos por aminorar las desigualdades entre los países (el poder). Este pensamiento, en cierta medida utópico, favoreció desconocimiento sistemático de los factores sociales, culturales y políticos involucrados en toda relación de cooperación, de este modo, las muchas veces contradictorias visiones del mundo, las diferencias culturales y los contenidos estratégicos de la cooperación no eran objeto de discusión y debate.

En ese sentido, hay que reconocer que la globalización implica la difusión internacional de valores en torno a los cuales hay una creciente aceptación y consenso: la equidad, los derechos humanos, el respeto a las minorías, la solidaridad son valores que sintetizan algunas de las aspiraciones comunes a las sociedades de las Américas (CEPAL, 2000).

Una condición indispensable tiene que ver con lo que podríamos llamar, inspirándonos en Giddens (1991), una actitud reflexiva de los actores involucrados en las relaciones de cooperación. Esto quiere decir que no existen los modelos definitivos ni formas de cooperación que no requieran una constante exploración crítica. Los actores participantes en una relación de CTI no sólo deben orientarse por las acciones de los demás actores sino que también deben dirigir su atención a las prácticas que regularmente se 
despliegan. Esta actitud, por un lado, permite revisar si los pasos que se dan están de acuerdo con el itinerario diseñado y, por otro lado, si son consistentes con los valores que sustentan nuestras prácticas.

La solidaridad enten dida como integración social, la equidad que aspira a reducir la desigualdad social y la eficiencia que busca dar sustentabilidad a los modelos de cooperación, son valores en torno a los cuales la CTI debería gravitar. Esto implica, por un lado, reconocer que las sucesivas reformas de los sistemas nacionales de salud en los países de Centro y Sudamérica arrojaron resultados dispares en términos sociales. $M$ ientras que en algunos casos las reformas condujeron a un serio deterioro de los principios de solidaridad y universalidad de los servicios, así como a una ampliación de la brecha de desigual dad entre los grupos sociales; en otras experiencias se ha logrado integrar estos principios en los nuevos esquemas de funcionamiento de los sistemas de salud (Sojo, 2000). La CEPAL conjetura que en los casos más radicales, como el de Chile, quizá sea necesario encarar unas "reformas de las reformas" (CEPAL, 2000).

Esto nos lleva a un segundo aspecto de esta relación, la actitud reflexiva debe evaluar las acciones de la cooperación técnica en función del impacto que las medidas tendrán en los niveles deintegración social, equidad y eficiencia a mediano y largo plazo. Esta preocupación no es menor, muchas veces la excesiva especialización técnica hizo menos evidente los efectos sociales a mediano y largo plazo de la cooperación técnica. La organización de la cooperación en torno a proyectos concretos también dificultaba la correcta apreciación de los resultados de la cooperación técnica. El interés por los efectos de las acciones complementa pero no se reduce a los objetivos de las evaluaciones programáticas.

Exigirle a la cooperación técnica internacional que integre estas dimensiones a su horizonte de práctica implica también modificar ciertos enfoques tradicionales de conceptuación de la integraciónn social, la equidad y la eficiência. En ese sentido, por ejemplo, el ingreso per capita de un grupo social pierde fuerza como indicador de los procesos sociales que interesa observar.

Al respecto, es importante evocar aquí dos conceptos centrales en la reflexión de los problemas del desarrollo, las nociones de capacidad y de capital social. Las nociones de capacidades y funcionamientos desarrolladas por A martya
Sen permitieron desplazar la atención desdelos medios para alcanzar el bienestar (vg.: ingresos) a los componentes del bienestar (los funcionamientos y las capacidades). Los funcionamientos pueden definirse como el nivel de bienestar de una persona en un momento determinado, está constituido por todos los elementos que definen ese bienestar (salud, educación, nutrición, empleo) y no pueden reducirse a una sola dimensión. La capacidad de funcionar se refiere a la libertad del individuo para elegir un tipo u otro de vida. Esto, por lo tanto, constituye la libertad de la persona (Sen, 1992 y 1999).

La cooperación técnica internacional tiene en este concepto un instrumento muy potente. Por un lado, las acciones en el campo de la salud tendrían como objetivo la expansión de las capacidades de las personas 0 , en palabras de Sen, ampliar la libertad de elección de los individuos. Las acciones en este caso no tienen como objeto inmediato proveer los medios para obtener determinado bien o servicio. Por otro lado, la expansión de las capacidades no es un proceso abstracto, se sustenta en la construcción de las instituciones y redes apropiadas para desarrollar el tipo de capacidades y los funcionamientos que se pretende alcanzar. La orientación a los medios (ingresos) o a las capacidades (libertad) explica que países con niveles de ingreso per capita bajos, como China o Costa Ri$\mathrm{ca}$, tengan indicadores de salud - mortalidad infantil - mucho mejores que países con niveles de ingreso más elevado, como Brasil o Sudáfrica (Sen, 1999).

El concepto de capital social ha sido ampliamente adoptado en los trabajos sobre desarrollo. Putnam sostiene que el capital social está constituido por redes sociales y normas de reciprocidad y confianza ¿Cuál es la relevancia del capital social para Putnam? U na sociedad que cuenta con una densa red de relaciones basadas en la reciprocidad es mucho más eficiente que una que carece de este atributo (Putnam, 2000; Kliksberg 1999). Coleman identifica tres formas de capital social, las obligaciones y expectativas que se forman a partir de la confianza el flujo de información que facilita un sistema social dado y las normas y sanciones que gobiernan un grupo social (1988). Las dos definiciones de capital social subrayan la importancia de aspectos que son decisivos para el individuo pero queno son atributo exclusivo de ellos. El capital social es un atributo del entorno social de los individuos que incide en las trayectorias individuales. 


\section{Temas de la Cooperación Técnica Internacional}

Quisiéramos concluir proponiendo algunos puntos para la discusión. Estos no pretenden agotar la extensa agenda de la cooperación internacional, sólo tienen el propósito de orientar la discusión hacia algunos de los tópicos que consideramos centrales para pensar la cooperación técnica en salud en la región.

- EL escenario institucional de la CTI es más complejo y heterónomo. Coexisten múltiples actores, proyectos y enfoques. La cooperación horizontal puede introducir cierta estabilidad por el lado de los países receptores de ayuda. Los organismos de CTI regionales pueden cumprir un papel muy efectivo organizando las relaciones de cooperación N orte-Sur.

- LaCTI horizontal debe también pensarse como mecanismo que permita contrarrestar las etapas de bajo compromiso de la cooperación N orte-Sur.

- Especialización técnica y visión integral. Sin afectar la calidad técnica de la cooperación, los organismos regionales e internacionales que ofrecen CTI deben contar con una visión integral de los problemas de salud. LaCTI en salud debe desarrolarse tomando en cuenta las acciones en pobreza, medio ambiente, educación, desarrollo urbano y empleo.
- Como parte de esa visión integral la CTI de be incorporar como objetivo prioritario el desarrollo de las capacidades institucionales y organizativas de los sistemas de salud previendo y evaluando, simultáneamente, las dificultades para mejorar tales capacidades

- La diferenciación territorial que acompaña al proceso de globalización requiere de organizaciones más flexibies. LaCTI en salud en las Américas debería incorporar paulatinamente mecanismos de organización basados en redes. La heterogeneidad regional del continente demanda la constitución de un enfoque que asegure coherencia en las perspectivas y flexibilidad en los enfoques.

- La CTI regional debe establecer un diálogo permanente con los actores globales, regionales y nacionales.

- LaCTI en salud debe desarrollar una estratégia para insertarse en los actuales procesos de integración sub-regional y regional.

- Evitar la selectividad y orientar la CTI regional al fortalecimiento de las capacidades institucionales.

- La CTI debe diseñar una forma de organización adecuada a los propósitos que se plantea. La introducción de formas de organización con base en redes permitirá alcanzar la flexibilidad que se requiere para trabajar en ambientes altamente diferenciados en cuanto a actores, problemáticas y diferencias regionales. 


\section{Referencias bibliográficas}

Abbasi K 1999. The World Bank and world health. Healtheare strategy. British M edical Journal 318.

Beck U 1998. ¿Q uê es la globalización? Falacias del globalismo, respuestas a la globalización. Paidos, Espanha.

Benn D 1996. South-South cooperation: a strategic dimension international development cooperation. Cooperation South Journal №l.

Berlinguer $\mathrm{G}$ 1999. Health and equity as a primary global goal. In International roundtable on responses to globalization: rethinking equity and health organization by Society for Intemational Development. World Health Organization and The Rockefeller Foundation.

Bobadilia J \& Cristina P 1992. H ow the epidemiological transition affects health policy issues in three Latin American countries. World Bank, Population, H ealth, and Nutrition, W orking Paper 987.

Braveman P, Barbara S\& H Jack G 2001. W orld H ealth Report 2000: how it removes equity from the agenda for public health monitoring and policy. British M edical Journal 323.

Castelis M 1998. Information technology, globalization and social development. Documento preparado para UN RISD Conference on Information Technologies and Social Development, Palais des Nations, Geneva, 2224 June 1998.

Castells M 1999. La era de la información: economia, sociedad y cultura. Vol. 1: La sociedad red. Siglo XXI, México.

Centro Latinoamericano de Investigacián en Sistemas de Salud 2001. Health promotion and disease prevention In southern cone countries. Final Report. Santiago, Chile.

Centro Latinoamericano de Investigación en Sistemas de Salud 2001. Vida sana es más vida. Enfrentando el desafio de la salud en el siglo XXI. Santiago, Chile.

CEPAL 2000. Equidad, desarrollo y ciudadania. Naciones Unidas, Comisión Económica para América Latina, Santiago de Chile.

Coleman J 1988. Social capital in the creation of human capital. American Journal of Sociology 94 Issue Supplement.

Costello A \&Alimuddin Z 2000. M oving to research partnerships in developing countries. British Medical Joumal 321.

Chambers R 1985. Shortcut methods of gathering social information for rural development projects. In M Cernea (ed.). Putting people first. Sociological variables in rural development. The World Bank, Oxford University Press.

Dachs N 2001. Inequidades en salud: cámo estudiarias. In H M álaga \& H Restrepo (eds.). Promoción de la salud: cómo construir vida saludabie. Editorial M édica Panamericana, Colombia.

Daulaire N 1999. Globalization and health. In Intemational roundtable on responses to globalization: rethinking equity and health organization by Society for International D evelopment. World Health Organization and The Rockefeller Foundation.

Díaz Polanco J 2001. El papel del financiamiento en los procesos de reforma del sector salud. In La reforma de salud de Venezuela. CENDES-CIID-M SDS-CORPOSALUD-IESA-FUNDACION POLAR, Caracas.

Fennei M \& Jeffrey A 1993. Perspectives on organizational change in the US medical care sector. Annual Review of Sociology 19:89-112.
Ferguson J 1994. The anti-politics machine. Development of the politization and bureaucratic power in Lesotho. University of M innesota Press.

Frenk J, Jaime S, Octavio GD, M ichael M cG \& Felicia K 1997. The future of world health: the new world order and international health. British M edical Joumal 314.

Giddens A 1991. La constitución de la sociedad. Bases para la teoria de la estructuración. Amorrortu Editores, Argentina.

Godlee F 1994. WHO in retreat: is it losing its influence? British M edical Journal 309.

Gwin, Catherine \& Joan N elson (eds.) 1997. Perspectives on aid and development. ODC Policy Essay № 22.

Harris E \& M areei T 2000. Health technology transfer. British M edical Journal 321.

Helleiner G 2000. Towards balance in aid relationship: donor performance monitoring in low-income developing countries. Cooperation South Journal 2.

IHSD 1998. Which health policies are pro-poor? Institute for Health Sector Development, Londres.

IOC (International Organizational Committee) 2000. Re port of the International conference on $\mathrm{H}$ ealth Research for Development. Bangkok, 10-15 October 2000.

Jacobs C 1996. Institutional strengthening and technical cooperation: developing a best practice model. IDS, Human Resources in Development Group Working Paper n. 5.

Kliksberg B 1999. Capital social y cultura, claves esenciales del desarrollo. Revista de la CEPAL 69.

M artin L 1992. Interest, power and multilateralism. International Organization 46(4).

M artínez J \& Liz C 1999. A review of human resource issues in the health sector. Briefing Paper, DFID Health Systems Resource Centre, Londres.

M ayorga R 1997. Chosing the gap. Inter-American Development Bank, Washington, DC.

M eyer C 1992. A step back as donors shift institutions building from the public to the "private" sector. World Developrnent 20(8).

Nathanson C 1996. Disease prevention as social change: toward a public health theory. Populations and Development Review 22(4).

Navarro V 2001. Thenew conventional wisdom: an evaluation of the WHO report. Health systems: improving performance. International J ournal of $\mathrm{H}$ ealth Services 31(1).

Nesiah D 1999. Some impacts of globalization on health and equity. In International roundtable on responses to globalization: rethinking equity and health organization by Society for International D evelopment. World $\mathrm{H}$ ealth Organization and The Rockefeller Foundation.

OPS 1992. Salud internacional. Un debate N orte-Sur. Serie de D esarrollo de Recursos H umanos 95.

OPS 1999. Orientaciones estratégicas y programáticas, 1999-2002. Documento Oficial 291. Washington, DC.

OPS 2001. Informe anual del Director. Organizacián Panamericana de la Salud, Washington DC.

PAHO 2001. Strategies for utilization of scientific information in decision-making for health equity. Division of Health and Human Development, Pan American $\mathrm{H}$ ealth Organization.

Panisset U 1992. Reflexiones acerca de la salud como asunto internacional. In Salud internacional. Un debate N orte-Sur. Organización Panamericana de la Salud, Serie de Desarrollo de Recursos H umanos № 95. 
Pérez B, Alfredo \& Iván S 1998. Cooperación técnica internacional. La dinámica y la experiencia mexicana. Secretaria de Relaciones Exteriores y M iguel Angei Porrúa Editor, M éxico.

PodoinyJ \& Karen P 1998. N etwork forms of organization. Annual Review of Sociology 24 (57-76).

Putnam R 2000. Bowling alone. The collapse and revival of American community. Touchstone Book, N ew York.

Rosanvalion P 1995. La nueva cuestión social. Repensar el Estado providencia. Ediciones Manantial, Buenos Aires.

Sachs 2000 . A new global consensus on helping the poorest of the poor. Conferencia en la Kennedy School of Government, $\mathrm{H}$ arvard U niversity, Cambridge, M assachusetts, M ay 15-16.

Sagasti F 1999. The shape of thing to come: development cooperation in the 21 st century. Cooperation South Journal 2.

Secretaría de Relaciones Exteriores 2000. La cooperación técnica entre países en desarrollo: mecanismos y perspectivas. Instituto M exicano de Cooperación Internacional, Secretaria de Relaciones Exteriores, M éxico.

Sen A 1992. Nuevo examen de la desigualdad. Alianza Editorial, Espanha.

Sen A 1999. Health in development. Bulletin of the World H ealth Organization $77(8)$.
Shaw $P$ 1999. N ew trends in public sector management in health. Applications in developed and developing countries. World Bank Institute, WBI Working Paper.

Sojo A 2000. Reformas de gestión en salud en América Latina: los cuasimercados de Colombia, Argentina, Chile y Costa Rica. Serie Políticas Sociales n 39, División de Desarrolio Social, CEPAL, Santiago de Chile.

Widdus R 2001. Public-private partnerships for health: their main targets, their diversity, and their future directions. Bulletin of the $\mathrm{W}$ orld $\mathrm{H}$ ealth O rganization 79(8).

Woodward D, Nick D, Robert B \& Debra L 2001. Globalization and health: a framework for analysis and action. Bulletin of the World $\mathrm{H}$ ealth Organization 79(9).

World Bank 1999. D evelopment effectiveness in health, nutrition, and population. Lessons from W orld Bank experience. Sector and thematic evaluations group, $\mathrm{Re}$ port № 19.266 .

W orld Bank 2001. World development report 2000-2001. Attacking poverty. Oxford U niversity Press, Washington.

World H ealth Organization 1999. The world health report 1999. M aking a difference. WHO, Geneva.

Artigo apresentado em 20/12/2002

A provado em 25/2/2003

Versão final apresentada em 17/3/2003 